\title{
ATENÇÃO ÀS NECESSIDADES HUMANAS BÁSICAS DO INDIVÍDUO COM AIDS
}

Priscila de Vasconcelos Monteiro', Maria Lúcia Duarte Pereira², Ana Ruth Macêdo Monteiro², Lúcia de Fátima da Silva², Maria Vilani Cavalcante Guedes², Fátima Dayanne Wirtzbiki Ferreira ${ }^{3}$

1Enfermeira. Mestre em Cuidados Clínicos em Enfermagem e Saúde. Universidade Estadual do Ceará. Fortaleza-CE-Brasil. Enfermeira. Doutora em Enfermagem. Universidade Estadual do Ceará. Fortaleza-CE-Brasil.

${ }^{3}$ Acadêmica. Universidade Estadual do Ceará. Fortaleza-CE-Brasil.

RESUMO: Objetivou-se com este estudo relatar a utilização da Sistematização da Assistência de Enfermagem elaborada a partir das Necessidades Humanas Básicas de indivíduo hospitalizado com Síndrome da Imunodeficiência Adquirida em dezembro de 2011. O método foi estudo de caso, que obteve aprovação do Comitê de Ética em Pesquisa; entre os diagnósticos de enfermagem, 11 determinavam necessidades fisiológicas, seis de segurança, quatro sociais, três de estima e um de autorrealização. A existência de necessidades não satisfeitas, como esperança e realização, prejudicou a motivação do indivíduo para satisfazer necessidades elementares, como as fisiológicas. Conclui-se que o processo de assistir deve ser planejado a partir das necessidades do indivíduo, pois é o centro das ações de enfermagem.

DESCRITORES: Cuidados de enfermagem; Teoria de enfermagem; Síndrome de imunodeficiência adquirida.

\section{ATTENTION TO THE BASIC HUMAN NEEDS OF THE INDIVIDUAL WITH AIDS}

\begin{abstract}
This study aimed to report the use of the Systematization of Nursing Care elaborated based on the Basic Human Needs of an individual hospitalized with Acquired Immunodeficiency Syndrome in December 2011. The method was a case study, which was approved by the Ethics Committee; among the nursing diagnoses, 11 specified physiological needs, six specified security needs, four specified social needs, three specified esteem needs, and one, self-actualization needs. The existence of unmet needs, such as hope and actualization, hindered the individual's motivation to meet elementary needs such as the physiological ones. It is concluded that the process of assisting must be planned, based on the individual's needs, as he or she is the center of the nursing actions.

DESCRIPTORS: Nursing care; Nursing theory; Acquired immunodeficiency syndrome.
\end{abstract}

\section{ATENCIÓN A LAS NECESIDADES HUMANAS BÁSICAS DEL INDIVIDUO CON SIDA}

RESUMEN: La finalidad de este estudio fue relatar la utilización da Sistematización de la Asistencia de Enfermería elaborada considerando las Necesidades Humanas Básicas de individuo hospitalizado con Síndrome de la Inmunodeficiencia Adquirida en diciembre de 2011. El método fue por estudio de caso, que obtuvo aprobación del Comité de Ética en Investigación; entre los diagnósticos de enfermería, 11 determinaban necesidades fisiológicas, seis de seguridad, cuatro sociales, tres de estima y un de autorrealización. La existencia de necesidades no satisfechas, como esperanza y realización, ha perjudicado la motivación del individuo en la satisfacción de necesidades fundamentales, como las fisiológicas. Se concluye que el proceso de asistir debe ser planeado a partir de las necesidades del individuo, pues es el centro de las acciones de enfermería.

DESCRIPTORES: Cuidados de enfermería; Teoría de enfermería; Síndrome de inmunodeficiencia adquirida.
Autor Correspondente:

Priscila de Vasconcelos Monteiro

Universidade Estadual do Ceará

Av. Paranjana, 1700 60.740-000 - Fortaleza-CE-Brasil

E-mail: privmonteiro@gmail.com
Recebido: 18/11/2012

Finalizado: 18/03/2014 


\section{INTRODUÇÃO}

Após quase 35 anos, desde a descrição dos primeiros casos, a Síndrome da Imunodeficiência Adquirida - aids já devastou milhões de vidas em todo o mundo. Compreende-se que as consequências do Vírus da Imunodeficiência Humana (HIV) geram necessidades que precisam ser satisfeitas. Desse modo, a equipe de enfermagem visa a atenuar o sofrimento, cooperar para a prevenção de agravos, promoção, recuperação da saúde e melhoria do bem-estar. Contudo, é indispensável que o profissional de enfermagem estabeleça um método para guiar a realização do atendimento(1).

O processo de enfermagem impõe-se como solução para organização das ações do enfermeiro e tem na Sistematização da Assistência de Enfermagem (SAE) um instrumento que o normatiza e auxilia sua aplicação. Por sua importância, o Conselho Federal de Enfermagem (COFEn) dispõe que a SAE seja estabelecida em todas as instituições de saúde em que são realizadas ações de enfermagem ${ }^{(2)}$. Para o uso da $\mathrm{SAE}$, uma possibilidade é norteá-la pela Teoria de Wanda Horta, a qual afirma que o homem busca, instintivamente, a satisfação de suas necessidades e os profissionais de enfermagem corroboram com a utilização de conhecimentos e princípios científicos para assistir o ser humano na satisfação de suas necessidades ${ }^{(3)}$.

Os conceitos da Teoria das Necessidades Humanas Básicas (NHB) de Wanda Horta permeiam o processo de enfermagem com observação, interação e intervenção junto ao indivíduo. A Teoria aponta cinco níveis de necessidades que precisam ser satisfeitos por ordem de importância. São eles: nível de necessidades fisiológicas; nível de necessidades de segurança; nível de necessidades sociais; nível de necessidades do ego ou estima e nível de necessidades de autorrealização ${ }^{(4)}$.

O referido processo de enfermagem foi proposto em seis etapas: histórico de enfermagem; diagnóstico de enfermagem; plano assistencial; plano de cuidados; evolução da enfermagem e prognóstico de enfermagem ${ }^{(3)}$. Diante do exposto, questionou-se de que forma o processo de enfermagem pode satisfazer as NHB de indivíduos com diagnóstico de aids. Objetivouse relatar a aplicação da SAE, elaborada a partir das NHB de um indivíduo hospitalizado com diagnóstico de aids.

\section{MÉTODO}

Estudo descritivo, do tipo estudo de caso, realizado em unidade de isolamento de um hospital referência para o tratamento de doenças infecciosas no Estado do Ceará - Brasil. Participou do estudo um indivíduo adulto com diagnóstico e manifestações clínicas de aids, escolhido aleatoriamente. Os dados foram coletados através de entrevista individual e de informações do prontuário. Utilizou-se um instrumento com questões referentes à história clínica e às NHB.

Aplicou-se a SAE com base na Teoria das $\mathrm{NHB}$ e no Processo de Enfermagem proposto por Horta $^{(3-4)}$. A coleta de dados ocorreu em dezembro de 2011. A análise dos dados foi realizada de forma descritiva. O estudo obedeceu aos critérios éticos do Conselho Nacional de Saúde que trata de pesquisa com seres humanos ${ }^{(5)}$. O projeto teve aprovação do Comitê de Ética em Pesquisa da Universidade Estadual do Ceará sob processo n. 11517349.

\section{RESULTADOS}

A primeira etapa do processo de enfermagem, o histórico, trouxe como resultados: paciente do sexo feminino, 27 anos, solteira, natural de Fortaleza, internada há duas semanas em isolamento com diagnóstico provisório de Tuberculose (TB) Ganglionar. Há seis anos com diagnóstico de aids e internação anterior por TB pulmonar. Abandonou o tratamento medicamentoso de ambas as patologias e, atualmente, faz uso de álcool, tabaco, crack e maconha.

$\mathrm{Na}$ segunda etapa foram identificados 25 diagnósticos de enfermagem, a partir da taxonomia North American Nursing Diagnosis Association (NANDA), Nursing Interventions Classification (NIC) e Nursing Outcomes Classification (NOC) (6). De tal modo, 11 desses classificados como determinantes das necessidades fisiológicas, seis de segurança, quatro sociais, três de estima e um de autorrealização. Para fins de estudo, foram selecionados cinco principais que representassem cada nível de NHB. A 
seleção foi feita pela possibilidade de maior impacto no quadro clínico situacional do indivíduo. As etapas do processo de enfermagem como diagnóstico, plano assistencial, plano de cuidados e evolução, encontram-se resumidas nos Quadros 1 a 5, conforme nível de NHB.

Quadro 1 - Resumo de etapas da Sistematização da Assistência de Enfermagem referentes às necessidades fisiológicas. Fortaleza-CE-Brasil, 2011

\begin{tabular}{|l|l|l|}
\hline Diagnóstico & Plano Assistencial / de Cuidados & Evolução \\
\hline Nutrição desequilibrada: menos que & Incentivar a aceitação da dieta; Orientar & Compreende necessidade \\
as necessidades corporais relaciona- & sobre importância do equilíbrio nutricio-- & de ganho ponderal; per- \\
das aos fatores biológicos e ingestão & nal para sua recuperação; Monitorar peso; & manece com aceitação \\
me-norqueasnecessidadescorporais. & Contar com apoio da equipe de nutrição. & parcial da dieta. \\
\hline
\end{tabular}

Quadro 2 - Resumo de etapas da Sistematização da Assistência de Enfermagem referentes às necessidades de segurança. Fortaleza-CE-Brasil, 2011

\begin{tabular}{|l|l|l|}
\hline Diagnóstico & Plano Assistencial / de Cuidados & Evolução \\
\hline Comportamento de saúde propen- & Conhecer os determinantes deste & Não demonstra aumen- \\
so a risco relacionado à eficiência & comportamento; Utilizar a comu- & To do interesse no auto- \\
pessoal reduzida e atitude negativa & nicação terapêutica; Contar com o & cuidado; Não reconhece \\
em relação aos cuidados de saúde. & apoio do psicólogo; Reconhecer as & existência de problemas \\
& potencialidades que o indivíduo per- & na manutenção da pro- \\
& cebe em sua atual condição de vida; \\
& pria saúde.
\end{tabular}

Quadro 3 - Resumo de etapas da Sistematização da Assistência de Enfermagem referentes às necessidades sociais. Fortaleza-CE-Brasil, 2011

\begin{tabular}{|l|l|l|}
\hline Diagnóstico & Plano Assistencial / de Cuidados & Evolução \\
\hline Interação social prejudicada rela- & Rever a história social com o in- & Não demonstra interesse \\
cionada ao isolamento terapêutico. & divíduo; Estimular o indivíduo a ver- & em fazer parte de um gru- \\
balizar sentimentos de desconforto & po; Relaciona o descon- \\
quanto à situação social; Recomendar & forto da hospitalização e \\
a realização de atividades de grupo ou condição de saúde à & da çaça em grupos de apoio. & $\begin{array}{l}\text { impossibilidade de aju- } \\
\text { a participaçãa a mãe; Demonstra in- } \\
\text { diferença em relação aos } \\
\text { demais membros da famí- } \\
\text { lia, inclusive aos filhos. }\end{array}$ \\
\hline
\end{tabular}

Quadro 4 - Resumo de etapas da Sistematização da Assistência de Enfermagem referentes às necessidades de estima. Fortaleza-CE-Brasil, 2011

\begin{tabular}{|l|l|l|}
\hline Diagnóstico & Plano assistencial / de Cuidados & Evolução \\
\hline Enfrentamento defensivo carac-ter- & Avaliar a capacidade de compreender a & Demonstra capacidade li- \\
izado por negação de problemas & situação atual; Determinar os mecanismos & mitada em compreender \\
ou fraquezas evidentes e recusa de & de enfrentamento utilizados; Utilizar co- & a própria situação; Utiliza \\
receber ajuda. & municação terapêutica; estimular detecção & mecanismos de enfrenta- \\
& e expressão de sentimentos; reconhecer os & mento como drogadição \\
& pontos fortes do indivíduo; Adotar atitude de & e fuga; Mostra animação \\
& aceitação e respeito; Encaminhar para acom- & no diálogo sobre seus \\
& panhamento cognitivo comportamental. & pontos fortes. \\
\hline
\end{tabular}


Quadro 5 - Resumo de etapas da Sistematização da Assistência de Enfermagem referentes às necessidades de autorrealização. Fortaleza-CE-Brasil, 2011

\begin{tabular}{|l|l|l|}
\hline Diagnóstico & Plano assistencial / de Cuidados & Evolução \\
\hline Risco de sen-timento de impotên- & Evidenciar os fatores causadores ou & Verbaliza sentimentos de \\
cia relacionado à doença e padrões \\
de enfrentamento inadequado. & contribuintes; Evidenciar a disponibili- & desesperança quanto ao \\
dade e utilização de recursos de apoio; & futuro e desistência de lu- \\
& Demonstrar esperança e estimular revi- & tar pela própria vida; Não \\
são de experiências bem sucedidas; en- & demonstra interesse em \\
& volver o indivíduo no planejamento do & utilizar a rede de apoio; \\
& próprio cuidado; Apoiar esforços para & Expressa sentimentos de \\
& adotar atitudes positivas e de esperança. & $\begin{array}{l}\text { incapacidade em mudar a } \\
\text { própria realidade pessoal. }\end{array}$ \\
\hline
\end{tabular}

A sexta etapa do processo de enfermagem diz respeito à definição do prognóstico de enfermagem, que significa a estimativa da capacidade do ser humano em atender suas necessidades básicas. Neste caso, o prognóstico identificado foi de dependência parcial para satisfação dos cinco níveis de necessidades, sendo que a prevalência está relacionada à condição de debilidade física.

\section{DISCUSSÃO}

De maneira geral, na taxonomia NANDA existem mais diagnósticos de enfermagem voltados aos primeiros níveis de necessidades em detrimento de daquelas subjetivas ou mais elevadas. No entanto, para cuidar de forma integral, as ações de enfermagem devem abranger de igual modo a todos os níveis. Nas necessidades fisiológicas, estavam presentes debilidades consequentes às manifestações da aids e de doenças oportunistas. A coinfecção HIV/TB, comum entre os que são acometidos pelo vírus, pode ser identificada no caso estudado. A TB é a maior causa de morte entre pessoas vivendo com HIV/aids, sendo a taxa de óbito na coinfecção de $20 \%{ }^{(7)}$. No mundo, algo próximo a 13\% dos casos de TB ocorre entre indivíduos vivendo com $\mathrm{HIV}^{(8)}$.

Dentre as necessidades de segurança, o baixo nível de escolaridade e a deficiência na compreensão de conceitos de higiene e saúde mostraram-se prejudiciais ao comportamento de busca de saúde, como a não adesão ao tratamento e vulnerabilidade a novas infecções, fato que legitima resultados de estudos da área ${ }^{(9)}$. Nesse caso, a equipe de enfermagem necessita contar com o auxílio da equipe multiprofissional para oferecer cuidado global ao indivíduo, alcançando-o em sua necessidade ${ }^{(6)}$.

As necessidades sociais encontradas relacionavam-se ao uso de drogas ilícitas e à debilidade física consecutiva ao adoecimento. Além de consequências sociais, o uso de álcool e outras drogas contribuem para a não adesão terapêutica $^{(10)}$. A respeito das necessidades de estima, a atitude defensiva foi constante. Entende-se, que dentre as consequências da doença, o medo da discriminação e a vergonha colaboram com a situação de isolamento e se colocam como um sério empecilho à promoção do acesso ao tratamento e ao acompanhamento de saúde ${ }^{(11)}$.

Ao abordar necessidades de autorrealização, questões sobre autoconceito e autovalorização foram compreendidas com dificuldade. A limitação em tratar assuntos subjetivos pôs-se como obstáculo ao atendimento dos últimos níveis de NHB através da SAE. De acordo com a Teoria das NHB, as necessidades elementares, como fisiológicas e de segurança, precisam ser satisfeitas prioritariamente para que motivem a busca pela satisfação dos demais níveis ${ }^{(4)}$. Entretanto, para o caso em estudo, necessidades não satisfeitas nos últimos níveis, como as de autorrealização e estima, interferiram negativamente no desejo em satisfazer as primárias.

Por fim, o referencial da Teoria das NHB de Wanda Horta ${ }^{(3)}$ foi necessária na elaboração de prioridades de atendimento. A identificação de necessidades por meio dos diagnósticos de enfermagem colaborou com o cuidado envolvido com o outro, partindo de suas queixas. Além disso, este cuidado é aceito por parte dos profissionais de enfermagem por ser algo intrínseco ao processo de trabalho do enfermeiro ${ }^{(12)}$. 


\section{CONSIDERAÇÕES FINAIS}

O processo de enfermagem proposto por Wanda Horta constituiu-se em instrumento essencial para a efetivação do cuidado de enfermagem de modo organizado e voltado para as NHB. Entretanto, dificuldades do indivíduo na compreensão de conceitos sobre estima e autorrealização se puseram como barreira para implementação da $\mathrm{SAE}$, nos níveis das necessidades mais subjetivas.

No sentido inverso à Teoria Motivacional de Maslow, necessidades não satisfeitas de esperança e realização extinguiram o desejo do indivíduo em satisfazer necessidades primordiais de alimentação, saúde e segurança. Desta forma, ouvir atentamente a fim de conhecer o indivíduo é o primeiro passo para reajustar os cuidados de atenção à saúde, de forma que o indivíduo seja o foco e não a rotina institucional ou o instrumento do processo de enfermagem.

Sugerem-se interações mais prolongadas entre profissionais e pacientes que possibilitem a existência de relacionamento terapêutico, vínculo e confiança, o que possibilitará ação mais efetiva no auxílio à satisfação das necessidades mais elevadas.

\section{REFERÊNCIAS}

1. Marques DKA. Construção e validação de um instrumento para a sistematização da assistência de enfermagem ao adolescente hospitalizado [Dissertação]. João Pessoa (PB): Universidade Federal da Paraíba; 2008.

2. Conselho Federal de Enfermagem. Resolução n. 358, de 15 outubro 2009. Dispõe sobre a Sistematização da Assistência de Enfermagem - SAE - nas instituições de saúde brasileiras. Rio de Janeiro: COFEN; 2009.

3. Horta WA. Processo de enfermagem. São Paulo: EPU; 1979.

4. Maslow AH. Motivación y personalidad. $1^{\text {a }}$ ed. Barcelona: Sagitario; 1954.

5. Ministério da saúde (BR). Conselho Nacional de Saúde. Diretrizes e normas regulamentadoras de pesquisas envolvendo seres humanos. Resolução n. 196, de 10 de outubro de 1996. Brasília; 1996.

6. Doengens ME, Moorhouse MF, Murr AC. Diagnósticos de Enfermagem: intervenções, prioridades, fundamentos. Rio de Janeiro: Guanabara Koogan; 2011.

7. Ministério da Saúde (BR). Manual de Recomendações para o Controle da Tuberculose no Brasil. Brasília: Ministério da Saúde; 2011. 284 p.

8. World Health Organization (WHO). Global Tuberculosis Control. Geneva: World Health Organization; 2011. $258 \mathrm{p}$.

9. Schilkowsky LB, Portela MC, Sá MC. Factors associated with HIV/AIDS treatment dropouts in a special care unit in the City of Rio de Janeiro, RJ, Brazil. Rev. bras. epidemiol. [Internet]. 2011;14(2) [acesso em 20 jan 2014]. Disponível: http://dx.doi.org/10.1590/S1415790X2011000200001

10. Padoin SMM, Zuge SS, Santos EEP, Primeira MR, Aldrighi JD, de Paula CC. Adesão à terapia antirretroviral para HIV/Aids. Cogitare enferm. [Internet]. 2013;18(3) [acesso em 21 jan 2014]. Disponível: http://ojs.c3sl. ufpr.br/ojs2/index.php/cogitare/article/view/33553

11. Rodrigues AS, Jesus MC, Silva LS, Oliveira JF, Paiva MS. Representações sociais de adolescentes e jovens vivendo com HIV acerca da adolescência, sexualidade e AIDS. Rev. Eletr. Enf. [Internet]. 2011;4(13) [acesso em 12 dez 2012]. Disponível: http://www.fen.ufg.br/ revista/v13/n4/pdf/v13n4a12.pdf.

12. Santana LL, Mazza VA, Taube SAM, Sarquis LMM. Diagnósticos e intervenções de enfermagem em unidade de pronto atendimento à luz das necessidades humanas básicas. Cogitare enferm. [Internet] 2011;16(4) [acesso em 06 dez 2012]. Disponível: http://ojs.c3sl.ufpr.br/ojs2/index.php/cogitare/article/ viewFile/25436/17057. 